\title{
Rapid Fluorescent Screening for Bifunctional Amine-Acid Catalysts: Efficient Syntheses of Quaternary Carbon Containing Aldols under Organocatalysis
}

Nobuyuki Mase†, Fujie Tanaka*, and Carlos F. Barbas, III*

The Skaggs Institute for Chemical Biology and the Department of Molecular Biology, The Scripps Research Institute, 10550 North Torrey Pines Road, La Jolla, California 92037

ftanaka@scripps.edu, carlos@scripps.edu.

\section{Supporting Information}

General. Chemicals and solvents were either purchased from commercial suppliers or purified by standard techniques. For thin-layer chromatography (TLC), silica gel plates Merck 60 F254 were used and compounds were visualized by irradiation with UV light and/or by treatment with a solution of phosphomolybdic acid in ethanol followed by heating. Flash column chromatography was performed using silica gel Merck 60 (particle size 0.040-0.063 mm). ${ }^{1} \mathrm{H}$ NMR and ${ }^{13} \mathrm{C}$ NMR spectra were recorded on Valian Inova-399 and Mercury-300. Chemical shifts are given in $\square$ relative to tetramethylsilane (TMS), the coupling constans $J$ are given in $\mathrm{Hz}$. The spectra were recorded in $\mathrm{CDCl}_{3}$ as solvent at room temperature, TMS served as internal standard $(\square=0 \mathrm{ppm})$ for ${ }^{1} \mathrm{H} \mathrm{NMR}$, and $\mathrm{CDCl}_{3}$ was used as internal standard $(\square=77.0)$ for ${ }^{13} \mathrm{C}$ NMR. High-resolution mass spectra were recorded on an Ion Spec Fourier Transform Mass Spectrometer using dihydrobenzoic acid (DHB) as the matrix.

The direct aldol reaction of $\square, \square$-dialkylaldehde 7 a with $p$-nitrobenzaldehyde (8a) (Table 1, entry 4): $p$-Nitrobenzaldehyde $(\mathbf{8 a}, 0.5 \mathrm{mmol})$ was dissolved in anhydrous DMSO $(0.5 \mathrm{~mL})$ and isobutyraldehyde (7a, $0.6 \mathrm{mmol})$ was added. To the mixture acetic acid $(0.125 \mathrm{mmol})$ and pyrrolidine $(0.025 \mathrm{mmol})$ were added. After stirring for $2 \mathrm{~h}$ at room temperature, the reaction mixture was directly purified by flash column chromatography ( silica gel $10 \mathrm{~g}$, hexanes/ethyl acetate $=70 / 30$ ) afforded the aldol product 9a ( $96 \%$ yield). 
The stereochemistry of 9d and 9e was deduced by ${ }^{1} \mathrm{H}$ NMR analysis according to the literature. See, Yamago, S.; Machii, D.; Nakamura, E. J. Org. Chem. 1991, 56, 2098-2106. The stereochemistry of 9f was determined by NOESY analysis after derivatization to 5-(4-isopropylbenzyl)-2,2,5-trimethyl-4-(4nitrophenyl)[1.3]dioxane.

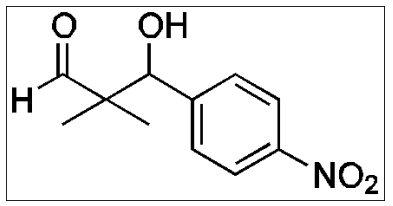

3-Hydroxy-2,2-dimethyl-3-(4-nitrophenyl)propionaldehyde (9a): $R_{\mathrm{f}}=0.24$ (hexane:AcOH = 70:30); ${ }^{1} \mathrm{H}$ NMR $(300 \mathrm{MHz}) \square=0.99\left(\mathrm{~s}, 3 \mathrm{H},-\mathrm{CH}_{3}\right), 1.05$ (s, $\left.3 \mathrm{H},-\mathrm{CH}_{3}\right), 2.97(\mathrm{~s}, 1 \mathrm{H},-\mathrm{OH}), 5.03(\mathrm{~s}, 1 \mathrm{H},-\mathrm{CHOH}), 7.48(\mathrm{~d}, J=8.4 \mathrm{~Hz}, 2 \mathrm{H})$, $8.18(\mathrm{~d}, J=8.4 \mathrm{~Hz}, 2 \mathrm{H}), 9.59$ (s, 1H, -CHO); ${ }^{13} \mathrm{C} \mathrm{NMR}(75 \mathrm{MHz}) \square=15.7,20.0,50.8,76.1,123.0$, 128.3, 146.8, 147.4, 205.6; MALDI-FTMS: Calcd for $\mathrm{C}_{11} \mathrm{H}_{13} \mathrm{NO}_{4}\left(\mathrm{MNa}^{+}\right)$: 246.0737, found: 246.0746; ESI-MS $224\left(\mathrm{MH}^{+}\right), 246\left(\mathrm{MNa}^{+}\right)$.

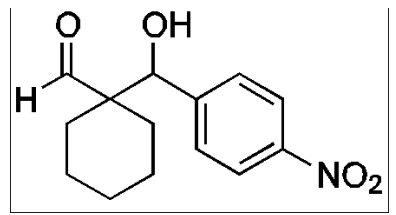

1-[Hydroxy-(4-nitrophenyl)methyl]cyclohexanecarbaldehyde (9b): $R_{\mathrm{f}}=$ 0.24 (hexane:AcOH = 70:30); ${ }^{1} \mathrm{H}$ NMR (400 MHz) $\square=0.80-1.40$ (m, 5H), $1.50-1.80(\mathrm{~m}, 3 \mathrm{H}), 1.90-2.10(\mathrm{~m}, 1 \mathrm{H}), 2.10-2.25(\mathrm{~m}, 1 \mathrm{H}), 2.60$ (brs, $1 \mathrm{H},-\mathrm{OH})$, $4.78(\mathrm{~s}, 1 \mathrm{H},-\mathrm{CHOH}), 7.43(\mathrm{~d}, J=8.8 \mathrm{~Hz}, 2 \mathrm{H}), 8.18(\mathrm{~d}, J=8.8 \mathrm{~Hz}, 2 \mathrm{H}), 9.72$ (s, 1H, -CHO); ${ }^{13} \mathrm{C}$ NMR (100 MHz) $\square=22.1,22.5,25.3,27.1,29.4,53.9,78.6,123.1,128.4,147.2$, 147.5, 207.9; ESI-MS $298\left([\mathrm{M}+\mathrm{Cl}]^{-}\right)$.

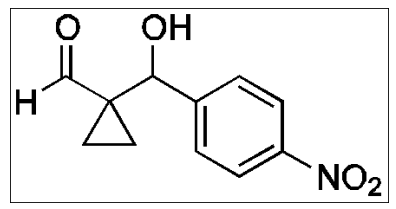

1-[Hydroxy-(4-nitrophenyl)methyl]cyclopropanecarbaldehyde $(9 \mathrm{c}): R_{\mathrm{f}}=$ 0.13 (hexane:AcOH = 70:30); ${ }^{1} \mathrm{H}$ NMR (400 MHz) $\square=0.94-1.06(\mathrm{~m} \mathrm{1H})$, 1.16-1.38 (m, 3H), $3.13(\mathrm{~d}, J=4.6 \mathrm{~Hz}, 1 \mathrm{H},-\mathrm{OH}), 5.24(\mathrm{~d}, J=4.6 \mathrm{~Hz}, 1 \mathrm{H},-$ $\mathrm{CHOH}), 7.57(\mathrm{~d}, J=8.8 \mathrm{~Hz}, 2 \mathrm{H}), 8.21(\mathrm{~d}, J=8.8 \mathrm{~Hz}, 2 \mathrm{H}), 8.77(\mathrm{~s}, 1 \mathrm{H},-\mathrm{CHO}) ;{ }^{13} \mathrm{C} \mathrm{NMR}(75 \mathrm{MHz}) \square$ $=10.1,11.8,37.7,71.3,123.3,127.5,147.3,147.6,201.0 ;$ ESI-MS $244\left(\mathrm{MNa}^{+}\right), 220\left([\mathrm{M}-\mathrm{H}]^{-}\right), 256$ $\left([\mathrm{M}+\mathrm{Cl}]^{-}\right)$.

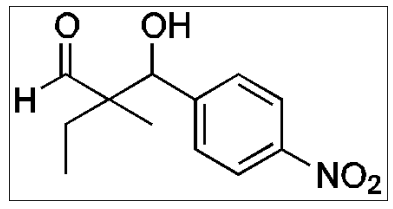

2-[Hydroxy-(4-nitrophenyl)methyl]-2-methylbutyraldehyde (9d): $R_{\mathrm{f}}=0.22$ (hexane:AcOH $=70: 30) ;{ }^{1} \mathrm{H}$ NMR $(300 \mathrm{MHz}$, diastereomer mixtures) $\square=0.80$ $0.95\left(\mathrm{~m}, 3 \mathrm{H},-\mathrm{CH}_{3}\right), 1.01$ (anti) and 1.05 (syn) (s, 3H, $\left.-\mathrm{CH}_{3}\right), 1.21-2.00(\mathrm{~m}$, $\left.2 \mathrm{H},-\mathrm{CH}_{2}\right), 2.80$ (brs, 1H, $\left.-\mathrm{OH}\right), 5.00$ (syn) and 5.08 (anti) (s, 1H, -CHOH), $7.48(\mathrm{~d}, J=8.7 \mathrm{~Hz}, 2 \mathrm{H})$, 8.16 (syn) and 8.18 (anti) (d, $J=9.0$ and $8.4 \mathrm{~Hz}, 2 \mathrm{H}), 9.63$ (anti) and 9.64 (syn) (s, 1H, -CHO); ESIMS $237\left(\mathrm{MH}^{+}\right)$. 


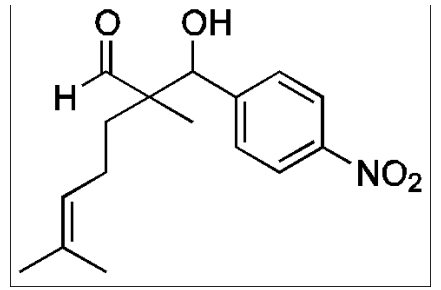

2-[Hydroxy-(4-nitrophenyl)methyl]-2,6-dimethylhept-5-enal (9e): $R_{\mathrm{f}}=$ 0.41 (hexane: $\mathrm{AcOH}=70: 30) ;{ }^{1} \mathrm{H}$ NMR $(400 \mathrm{MHz}$, diastereomer mixtures) $\square$ $=1.04$ (anti) and $1.06(\mathrm{syn})\left(\mathrm{s}, 3 \mathrm{H},-\mathrm{CH}_{3}\right), 1.10-1.75\left(\mathrm{~m}, 2 \mathrm{H},-\mathrm{CH}_{2}\right), 1.56(\mathrm{~s}$, $\left.3 \mathrm{H},-\mathrm{CH}_{3}\right), 1.66\left(\mathrm{~s}, 3 \mathrm{H},-\mathrm{CH}_{3}\right), 1.80-2.05\left(\mathrm{~m}, 2 \mathrm{H},-\mathrm{CH}_{2}\right), 2.86$ (brs, $1 \mathrm{H},-$ $\mathrm{OH}), 4.90-5.05(\mathrm{~m}, 1 \mathrm{H},-\mathrm{CH}=), 5.00$ (syn) and 5.09 (anti) (s, 1H, $-\mathrm{CHOH})$, 7.50 (d, $J=8.6 \mathrm{~Hz}, 2 \mathrm{H}), 8.20$ (d, $J=8.6 \mathrm{~Hz}, 2 \mathrm{H}), 9.66$ (anti) and 9.68 (syn) (s, 1H, -CHO); ESI-MS $292\left(\mathrm{MH}^{+}\right), 314\left(\mathrm{MNa}^{+}\right), 326\left([\mathrm{M}+\mathrm{Cl}]^{-}\right)$.

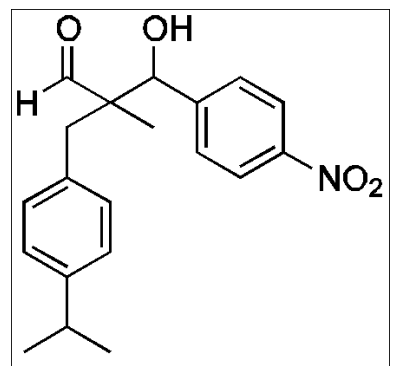

3-Hydroxy-2-(4-isopropylbenzyl)-2-methyl-3-(4nitrophenyl)propionaldehyde (9f): $R_{\mathrm{f}}=0.40$ (hexane: $\mathrm{AcOH}=70: 30$ ); ${ }^{1} \mathrm{H}$ NMR (300 MHz, diastereomer mixtures) $\square=0.97$ (anti) and 1.04 (syn) (s, 3H, $\left.-\mathrm{CH}_{3}\right), 1.21$ (syn) and 1.22 (anti) (d, $J=6.9$ and $\left.7.2 \mathrm{~Hz}, 6 \mathrm{H}, 2 \mathrm{X}-\mathrm{CH}_{3}\right), 2.54$ (syn) and 2.68 (anti) (d, $J=13.5$ and $13.5 \mathrm{~Hz}, 1 \mathrm{H}), 2.74-3.20$ (m, 2H, -CH, $\mathrm{OH}), 2.97$ (anti) and 3.38 (syn) (d, $J=13.5$ and $13.5 \mathrm{~Hz}, 1 \mathrm{H}), 4.97$ (anti) and 5.05 (syn) (s, 1H, -CHOH), 6.90-7.04 (m, 2H), 7.06-7.16 (m, 2H), 7.45 (anti) and 7.51 (syn) (d, $J=9.0$ and $8.7 \mathrm{~Hz}, 2 \mathrm{H}), 8.12-8.22(\mathrm{~m}, 2 \mathrm{H}), 9.54$ (syn) and 9.72 (anti) (s, 1H, -CHO); MALDI-FTMS: Calcd for $\mathrm{C}_{20} \mathrm{H}_{23} \mathrm{NO}_{4}\left(\mathrm{MNa}^{+}\right)$: 364.1519, found: 364.1515 .

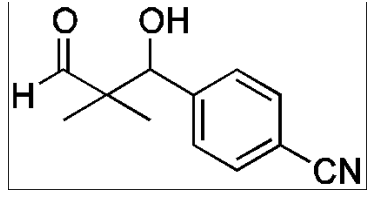

3-Hydroxy-2,2-dimethyl-3-(4-cyanophenyl)propionaldehyde (9g): $R_{\mathrm{f}}=0.20$ (hexane: $\mathrm{AcOH}=70: 30) ;{ }^{1} \mathrm{H}$ NMR $(400 \mathrm{MHz}) \square=0.96\left(\mathrm{~s}, 3 \mathrm{H},-\mathrm{CH}_{3}\right), 1.04$ (s, $\left.3 \mathrm{H},-\mathrm{CH}_{3}\right), 3.80$ (brs, $\left.1 \mathrm{H},-\mathrm{OH}\right), 4.97$ (s, $\left.1 \mathrm{H},-\mathrm{CHOH}\right), 7.44(\mathrm{~d}, J=8.4 \mathrm{~Hz}, 2 \mathrm{H})$, $7.63(\mathrm{~d}, J=8.4 \mathrm{~Hz}, 2 \mathrm{H}), 9.62$ (s, $1 \mathrm{H},-\mathrm{CHO}) ;{ }^{13} \mathrm{C} \mathrm{NMR}(100 \mathrm{MHz}) \square=15.5,19.7,50.7,76.3,111.5$, 118.6, 128.2, 131.6, 145.1, 205.9; ESI-MS 202 ([M-H]'), 238 ([M+Cl] $]^{-}$. 

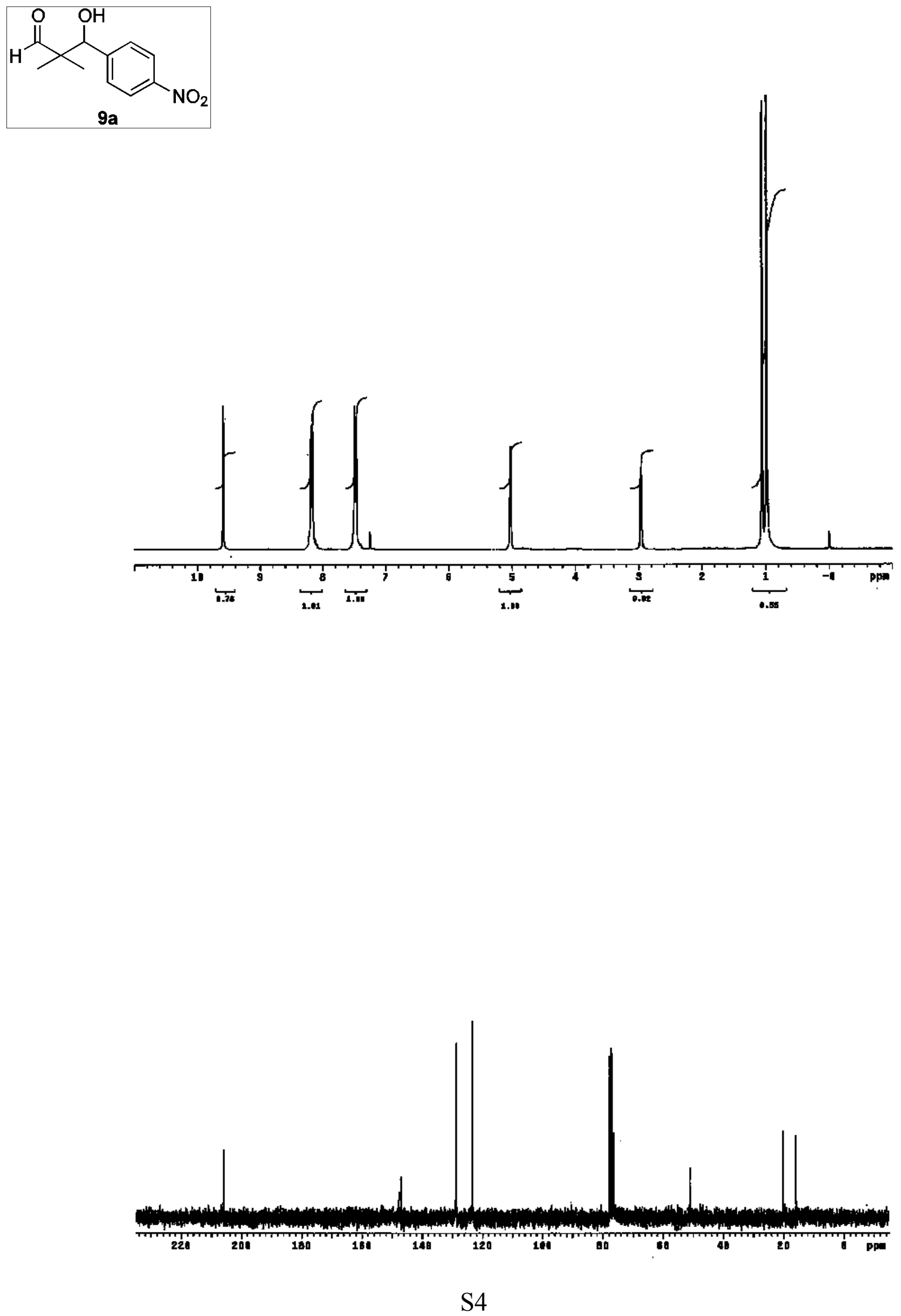

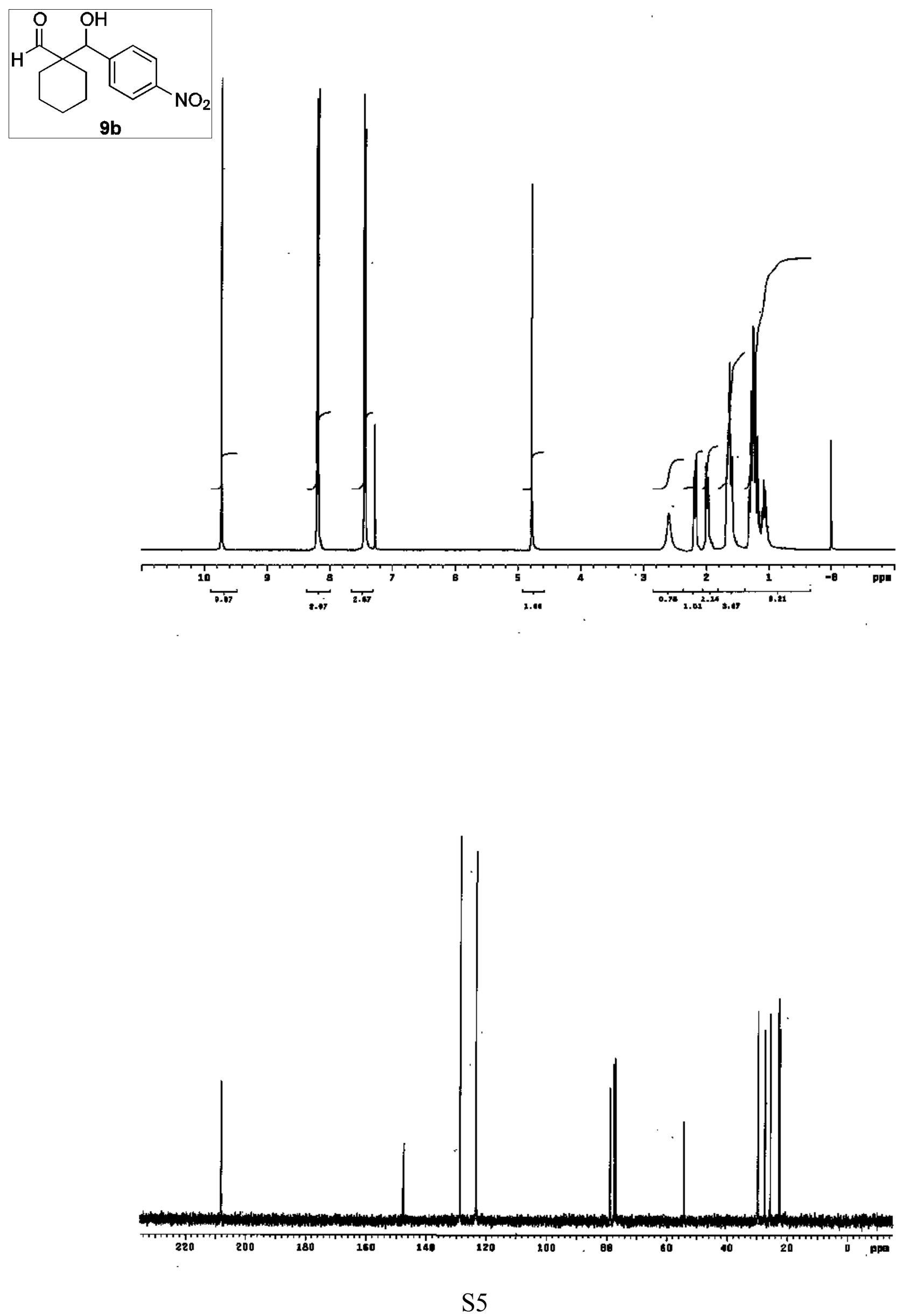

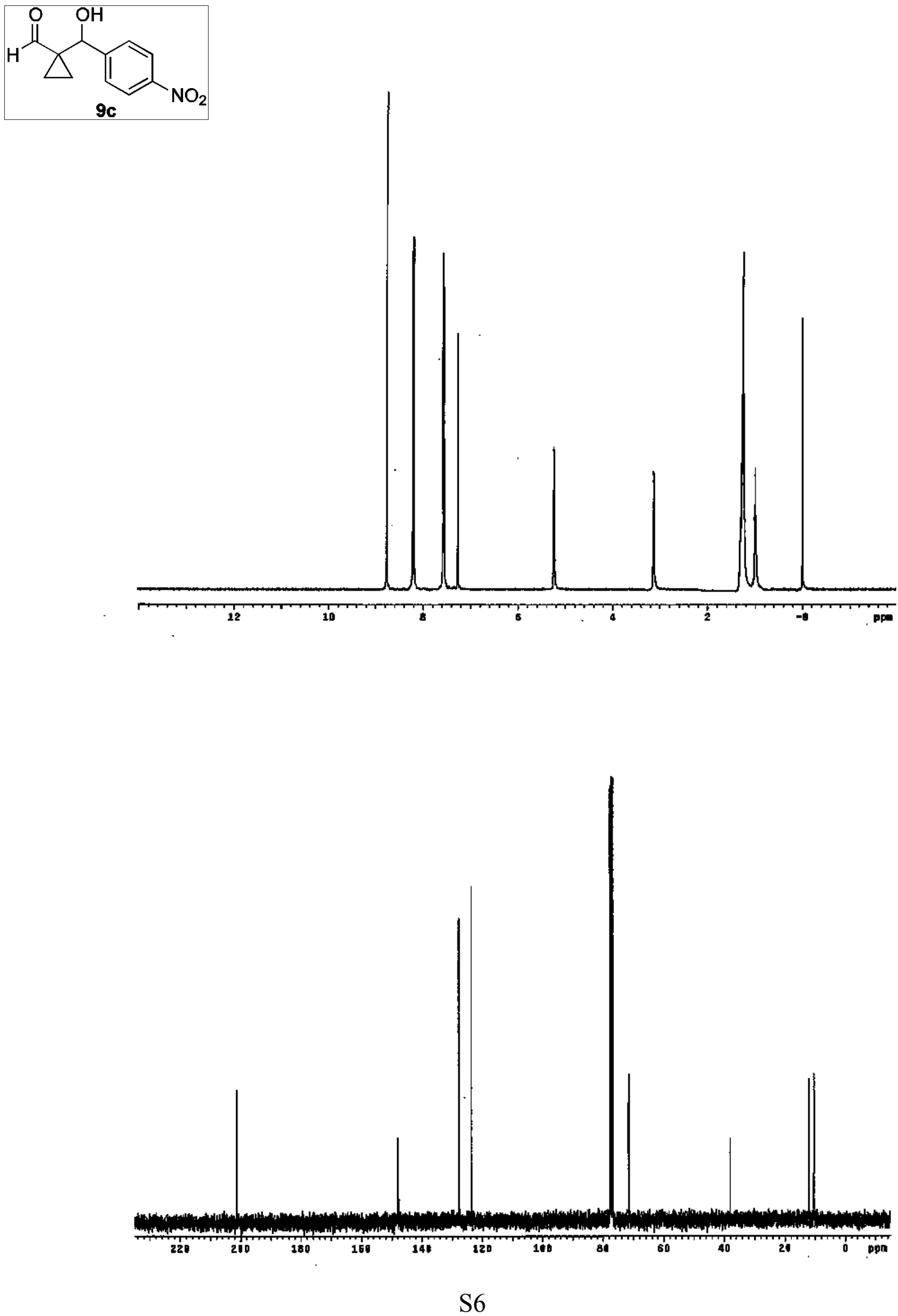

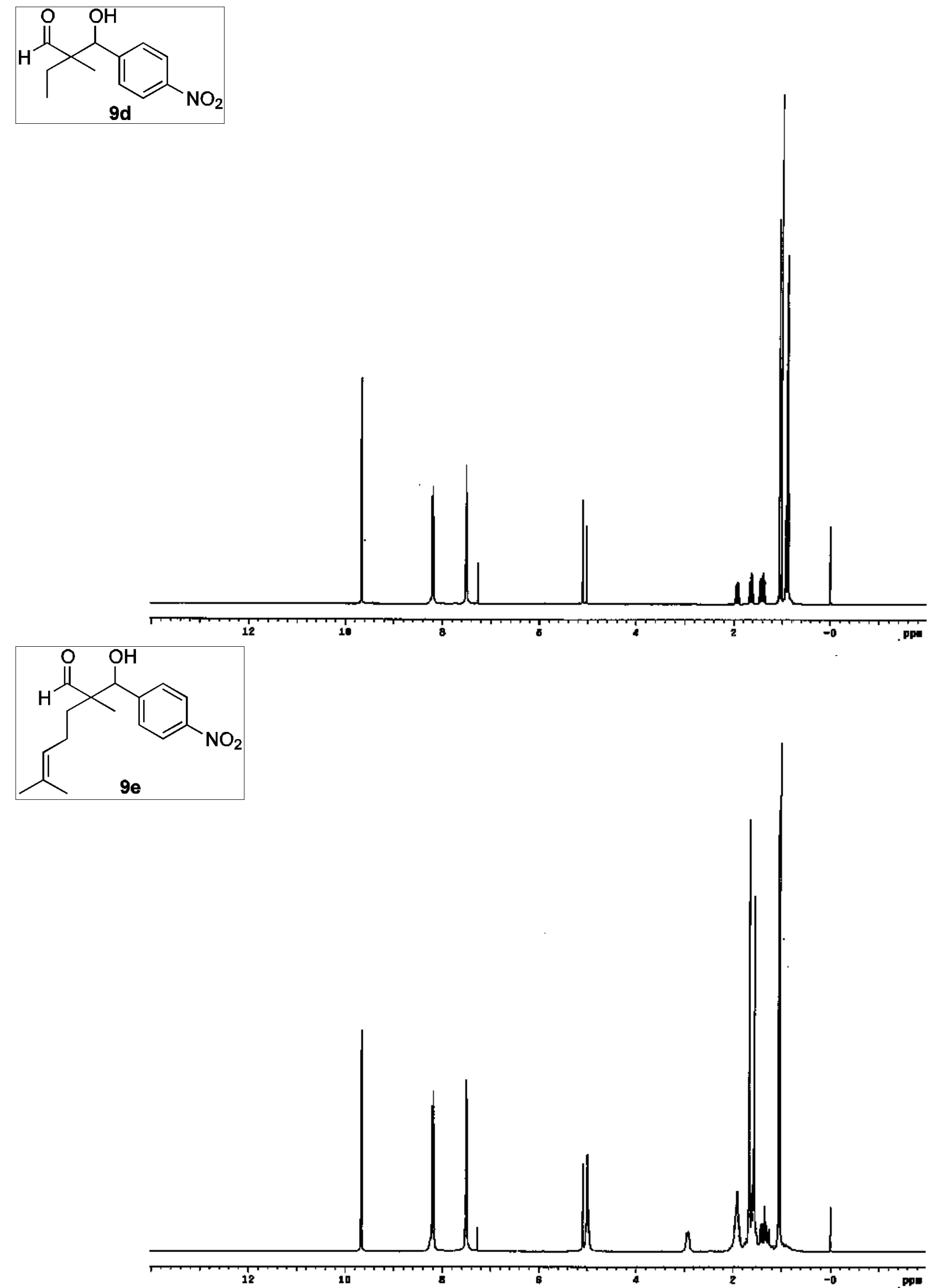

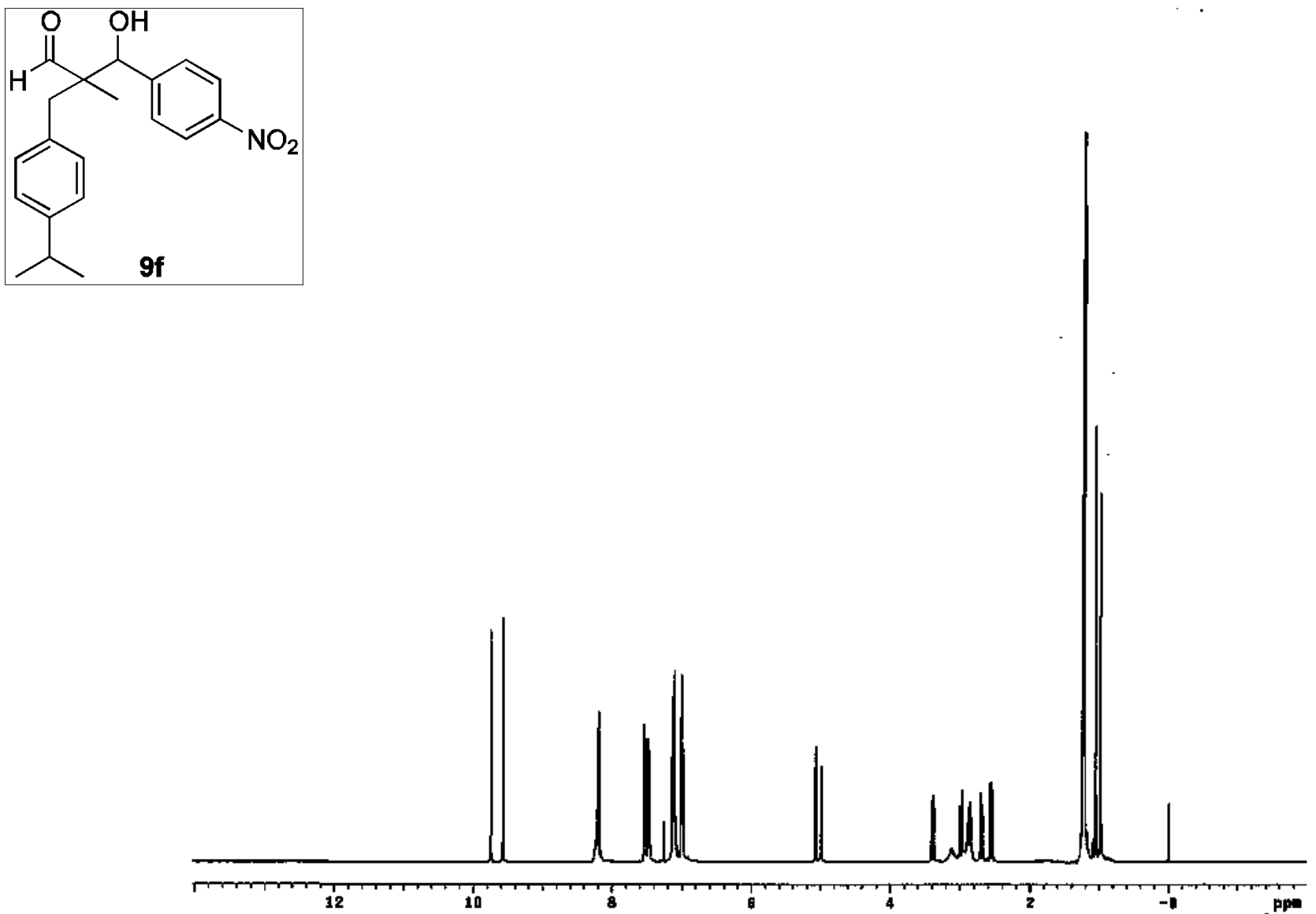

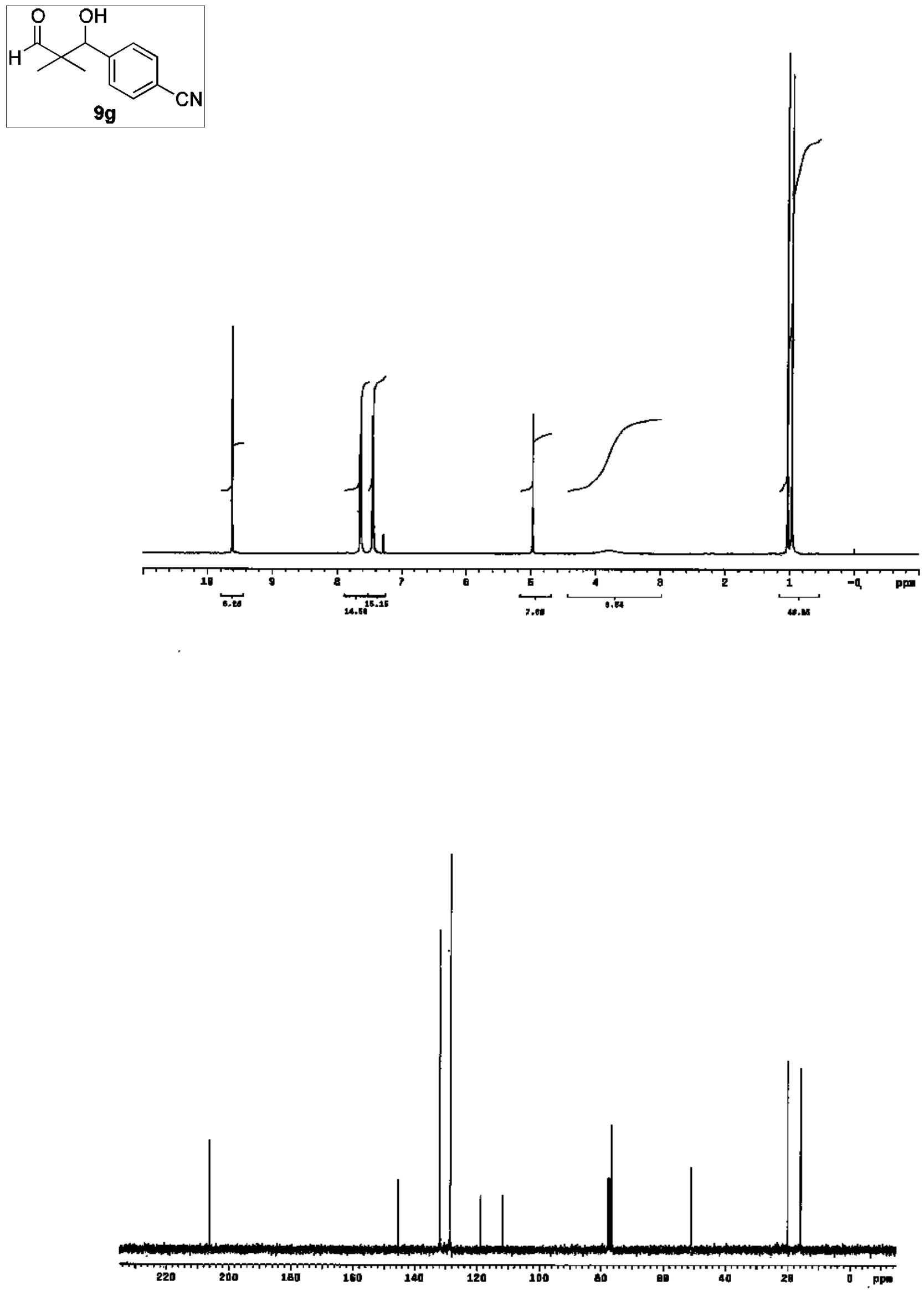

S9 